Giovanna Fabio: None declared, Laura Obici: None declared, Maria Alessio: None declared, Donato Rigante: None declared, Luca Cantarini: None declared, Antonella Insalaco: None declared, Marco Cattalini: None declared, Maria Cristina Maggio: None declared, Gabriele Simonini: None declared, Alma Nunzia Olivieri: None declared, Serena Pastore: None declared, Maddalena Lancieri: None declared, Nicolino Ruperto Grant/research support from: Bristol-Myers Squibb, Eli Lily, F Hoffmann-La Roche, GlaxoSmithKline, Janssen, Novartis, Pfizer, Sobi (paid to institution), Consultant of: Ablynx, AbbVie, AstraZeneca-Medimmune, Biogen, Boehringer Ingelheim, Bristol-Myers Squibb, Eli Lily, EMD Serono, GlaxoSmithKline, Hoffmann-La Roche, Janssen, Merck, Novartis, Pfizer, R-Pharma, Sanofi, Servier, Sinergie, Sobi, Takeda, Speakers bureau: Ablynx, AbbVie, AstraZeneca-Medimmune, Biogen, Boehringer Ingelheim, Bristol-Myers Squibb, Eli Lily, EMD Serono, GlaxoSmithKline, Hoffmann-La Roche, Janssen, Merck, Novartis, Pfizer, R-Pharma, Sanofi, Servier, Sinergie, Sobi, Takeda, Marco Gattorno Consultant of: Sobi, Novartis, Speakers bureau: Sobi, Novartis

DOI: 10.1136/annrheumdis-2020-eular.1402

\section{OP0274 CLINICAL ASPECTS, LABORATORY CHARACTERISTICS AND TREATMENT RESPONSES OF AA AMYLOIDOSIS: SINGLE CENTER EXPERIENCE WITH 163 PATIENTS}

M. Bektas ${ }^{1}$, N. Koca ${ }^{1}$, E. Oguz2, B. Ince ${ }^{1}$, Y. Yalçınkaya ${ }^{1}$, B. Artim-Esen ${ }^{1}$, M. Inanc ${ }^{1}$, M. L. Ocal' ${ }^{1}$, A. Gül' ${ }^{1}{ }^{1}$ Istanbul Faculty of Medicine, Internal Medicine, Rheumatology Division, Istanbul, Turkey; ${ }^{1}$ Istanbul Faculty of Medicine, Internal Medicine, Rheumatology Division, Istanbul, Turkey

Background: AA amyloidosis has been associated with uncontrolled chronic inflammatory diseases such as rheumatoid arthritis (RA), ankylosing spondylitis (AS), inflammatory bowel disease (IBD) and hereditary periodic fever syndromes, and the most common cause is familial Mediterranean fever (FMF) in Turkey. Objectives: We herein aimed to evaluate clinical and laboratory characteristics and treatment responses of patients with AA amyloidosis retrospectively in a tertiary referral center.

Methods: Study group was consisting of patients with biopsy proven AA amyloidosis, and their data were recorded from their charts. Treatment responses were categorized as follows: complete response was defined as no increase in serum creatinine and a proteinuria below $1 \mathrm{gr} /$ day; partial response as $50 \%$ decrease in proteinuria; and stable disease as no significant change in serum creatinine and proteinuria. Progressive disease was defined as increase in serum creatinine and/or proteinuria under treatment.

Results: 173 patients were identified, and 10 patients with no biopsy result and/ or missing data were excluded. A total of 163 patients (79 females, 84 males) were included in the study. Median age of patients was 45.4 , and median age at diagnosis of amyloidosis was 33.5. Most common cause of amyloidosis was FMF (78.5\%), followed by idiopathic cases $(7.9 \%)$ and patients with AS (4.9\%). A quarter (26\%) of amyloidosis patients had a family history for AA amyloidosis, and $59 \%$ of patients with FMF had a family history of FMF. Amyloidosis was confirmed by renal biopsy in $76.1 \%$, by gastrointestinal (GIS) biopsy in $11.7 \%$, and by other biopsies in the remaining. Renal involvement was documented in 160 (98.2\%) patients, while GIS involvement in $20.9 \%$, heart in $13.5 \%$, thyroid in $3.7 \%$ and bone marrow in $3.1 \%$. In FMF patients, most common MEFV mutation was M694V (77.7\%); and $66.7 \%$ of the patients had homozygous, $14.6 \%$ had compound heterozygous, and $18.7 \%$ heterozygous exon 10 variants. Mean age at diagnosis of amyloidosis was earlier in homozygotes (29.1) and compound heterozygotes (32.3) compared to heterozygotes (43.9) $(p=0.001)$. There was no difference in treatment responses, organ involvement, progression to end stage renal disease (ESRD) and mortality between monoallelic and biallelic exon 10 mutations $(p=0.42)$. While $44.3 \%$ ( $n$ $=70$ ) of patients had chronic renal disease (CRD) at time of diagnosis, ESRD developed in $45.3 \%(n=73)$ of patients. During follow-up, 55 patients underwent renal transplantation and recurrence of renal amyloidosis occurred in $24 \%$ of them. Mean creatinine and proteinuria levels at time of diagnosis were higher in patients with ESRD than those without ESRD ( $p<0.001, p=0.03$ respectively). Progression to ESRD was significantly higher in patients with GFR $\leq 60 \mathrm{ml} / \mathrm{min}$ at time of admission ( $\% 14.5$ vs $\% 41.7, p=0.005$, Figure 1$)$. A total of $113(70.2 \%)$ patients used biological agents, most commonly used biological agent was anakinra $(n=81)$. Canakinumab was used in 17 and other biological agents in 17 patients. Complete response was observed in $49.1 \%$, partial response was observed in $6.2 \%$, and progressive progression was observed in $21.7 \%$. GIS and cardiac involvements were associated with progressive course $(p<0.001)$ and increased mortality $(p=$ $0.002, p<0.001$, respectively), and overall mortality rate was $8.7 \%$.

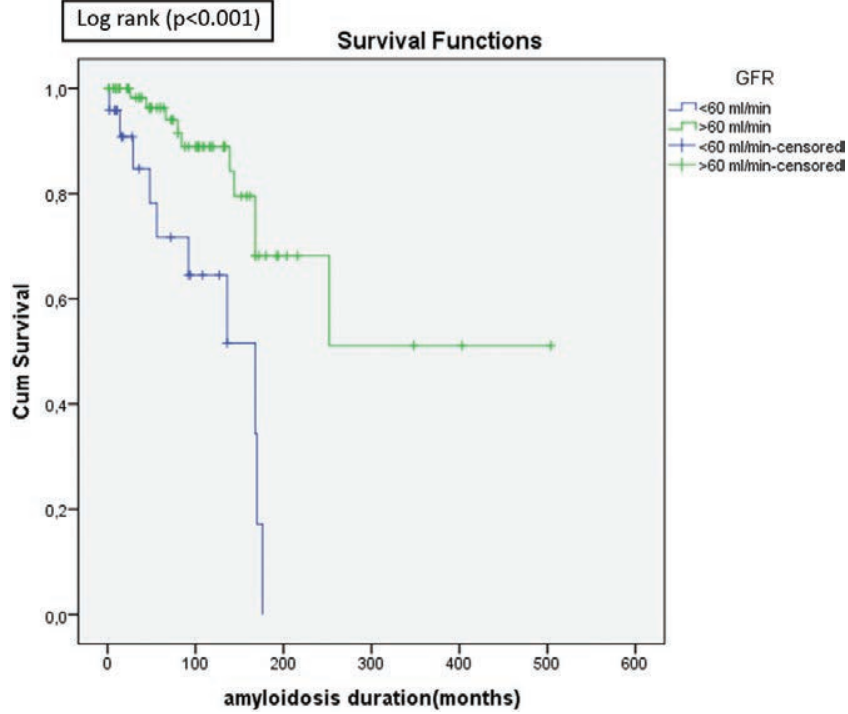

Figure 1 : Survival graphic of AA amyloidosis patients who developed ESRD according to their baseline GFR status

Conclusion: Increased rate of ESRD and progression of amyloidosis findings in patients who presented with GFR $<60 \mathrm{ml} / \mathrm{min}$ emphasize the importance of early diagnosis. Although mortality rate is very high in patients with $A A$ amyloidosis due to FMF disease, it may be possible to reduce mortality with an effective treatment. Disclosure of Interests: None declared

DOI: 10.1136/annrheumdis-2020-eular.3805

\section{OP0275 \\ REAL-WORLD CLINICAL BURDEN AND GLUCOCORTICOID USE IN PATIENTS WITH GIANT CELL ARTERITIS}

R. Punekar ${ }^{1}$, P. Lafontaine ${ }^{2}$, J. H. Stone ${ }^{3} .{ }^{1}$ Sanofi, Cambridge, United States of America; ${ }^{2}$ Sanofi, Bridgewater, United States of America; ${ }^{3}$ Harvard Medical School, Boston, United States of America

Background: Giant cell arteritis (GCA) is a rare form of vasculitis usually manifesting in people aged $\geq 50 \mathrm{yr}$ and is more common in women. Symptoms include headache, jaw claudication, fatigue, polymyalgia; and blindness if untreated. While risks of complications can be reduced with promptly administered highdose glucocorticoids (GC; $20-60 \mathrm{mg}$ for $2-4 \mathrm{wk}$, then slowly tapered), further risks of high GC exposure and related complications over the course of therapy remain.

Objectives: To compare GC use and GC-related complications in GCA patients (pts) vs a general population (GnP) cohort.

Methods: This retrospective, observational cohort study was based on Optum's de-identified Clinformatics ${ }^{\circledR}$ Data Mart Database (01/01/06-30/06/18, study period). The GCA cohort included pts with $\geq 1$ inpatient or $\geq 2$ outpatient claims $\geq 30$ days apart with GCA-related diagnosis codes (ICD-9: 446.5x/ICD-10: M31.6x) between 01/01/06-30/06/17 (pt identification period) during which first occurrence of a GCA-related medical claim was set as index date (ID). The GnP cohort included pts without any medical claims for rheumatoid arthritis, GCA or polymyalgia rheumatica diagnosis codes during the study period, with their ID set as 12 mo from start of continuous health plan enrollment. Pts in both cohorts were required to be age $\geq 50 \mathrm{yr}$ (on the ID) with continuous health plan enrollment $\geq 12$ mo pre- and post-ID. Cohorts were 1:1 propensity score matched. GC use and incidence of GC-related complications were assessed from GC initiation, starting from the baseline period (12-mo pre-ID) to the end of GC use during the post-index period (ie the end of data availability, end of the study period, or death, whichever occurred first). Descriptive analyses included mean, standard deviation (SD) and median values for continuous variables, and frequency ( $\mathrm{n}$ and \%) for categorical variables. Continuous variables were compared between matched cohorts using $t$-tests and Wilcoxon sum rank tests. Categorical variables were compared between matched cohorts using Chi-square tests or Fisher's exact tests. Duration of GC use was analyzed using the Kaplan-Meier method and compared between matched cohorts using log-rank tests.

Results: There were 6071 pts included in each of the GCA and matched GnP cohorts; median age per cohort was $76 \mathrm{yr}$, median Elixhauser comorbidity index score was 3.0, and the majority $(\sim 75 \%)$ were women. The median follow-up duration was 44 and 48 mo in the GCA and GnP cohorts, respectively. A higher proportion of pts in the GCA cohort than the GnP cohort ( 90.6 vs $63.8 \% ; p<0.001$ ) used GC. The mean (SD) duration of GC therapy was $230.5( \pm 326.8)$ days in 
the GCA cohort vs $36.3( \pm 107.2)$ days in the GnP cohort $(p<0.001)$. Although the mean (SD) daily dose of GC (prednisone equivalent) was similar in both cohorts (27.6 $[ \pm 28.20]$ vs $27.7[ \pm 25.18] \mathrm{mg})$, the mean $(\mathrm{SD})$ cumulative GC dose was significantly higher in the GCA cohort than the GnP cohort $(3503.0( \pm 4622.6) \mathrm{mg}$ vs 503.7 ( \pm 1593.51$) \mathrm{mg} ; p<0.001)$. This indicates that GCA pts had chronic GC exposure over the study period while GnP pts likely utilized higher dose GC burst therapy less frequently. The number of incident complications associated with GC use were significantly greater in the GCA cohort, and included hypertension, diabetes, skin toxicity, infections, neuropsychiatric effects, gastrointestinal complications, ocular effects, and cardiovascular disease $(p<0.05)$.

Conclusion: The overall GC burden in pts with GCA is significantly higher than the general population and may result in downstream complications related to GC exposure. The incidence of GC-related complications was statistically significantly higher in GCA pts compared with GnP pts, even with a short duration of GC use. The early onset of these complications may be a significant contributor to long-term healthcare costs in GCA pts

Acknowledgments: Study and medical writing (provided by Gauri Saal, MA, Economics, Prime, Knutsford, UK, under the direction of authors) were funded by Sanofi, Inc.

Disclosure of Interests: Rajeshwari Punekar Shareholder of: Sanofi, Employee of: Sanofi, Patrick LaFontaine Shareholder of: Sanofi, Employee of: Sanofi, John H. Stone Grant/research support from: Roche, Consultant of: Roche DOI: 10.1136/annrheumdis-2020-eular.4263

\section{OP0276 \\ CLINICAL PATTERNS AND FOLLOW-UP OF INFLAMMATORY ARTHRITIS AND OTHER IMMUNE- RELATED ADVERSE EVENTS INDUCED BY CHECKPOINT INHIBITORS. A MULTICENTER STUDY}

J. A. Gómez-Puerta ${ }^{1}$, C. Perez-Garcia ${ }^{2}$, D. Lobo Prat ${ }^{3}$, R. Gumucio ${ }^{1}$, F. Ojeda ${ }^{2}$, A. M. Millán Arciniegas ${ }^{3}$, S. Rodriguez Garcia ${ }^{1}$, V. Ruiz ${ }^{1}$, H. Corominas ${ }^{3}$. ${ }^{1}$ Hospital Clinic, Rheumatology, Barcelona, Spain; ${ }^{2}$ Hospital del Mar, Rheumatology, Barcelona, Spain; ${ }^{3}$ Hospital de la Santa Creu i Sant Pau, Rheumatology, Barcelona, Spain

Background: Immune checkpoint inhibitors (ICI), such as anti-CTLA-4and anti-PD1/PD-L1 monoclonal antibodies, have produced impressive clinical results in different types of cancer. However, immune-related adverse events (irAEs) may develop a wide spectrum of disabling syndromes. Knowledge of different rheumatic irAEs induced by $\mathrm{ICl}$ is increasing over the last years, however clinical patterns, time to onset of different irAEs according to treatment and follow-up are less well known.

Objectives: To describe different clinical patterns of rheumatic irAEs induced by $\mathrm{ICl}$ and their rheumatic and oncologic outcomes.

Methods: We included consecutive patients with rheumatic irAEs from 3 different referral centers in Barcelona with special emphasis in articular irAEs. Four main clinical syndromes were identified: inflammatory arthritis (IA), non-inflammatory arthralgias (NIA), psoriatic arthritis (PsA)-like and polymialgia (PMR)-like. We conducted a baseline visit and then follow-up in order to determine their clinical pattern, treatment response and outcome. Longitudinal visits were done from January 2017 to January 2020. Patients with other non-articular diagnosis were not included in the follow-up analysis.

Results: We included 55 patients. A total of 34 patients were male $(61.8 \%)$ with a mean age of $65.0 \pm 11.4$ years. Oncologic underlying diagnosis was lung carcinoma in $24(43.6 \%)$ patients, followed by melanoma in $17(29 \%)$, urothelial cancer in 4 (7.3\%), breast in $2(3.6 \%)$ and $2(3.6 \%)$ acute myeloid leukemia among others. Seven $(12.7 \%)$ patients received $\mathrm{ICl}$ as combined therapy. Different $\mathrm{ICI}$ were used including: Pembrolizumab in 21 (38.2\%), Nivolumab 13 (23.6 \%), Atezolizumab 6 (10.9\%), Nivolumab + ipilimumab $5(9.0 \%)$, Durvalumab $3(5.5 \%)$, Pembrolizumab + epacadostat in $2(3.6 \%)$, 2 anti TIM3, Atezolizumab+ Ibatasertib, Avelumab and Ipilimumab in one case each. 12 out of 55 patients had an underlying rheumatic disease before $\mathrm{ICI}$ treatment. Eleven patients developed other irAEs before or at the same time as rheumatic syndromes (mainly colitis and thyroiditis). Main rheumatic irAE included: IA in $23(41.8 \%)$, NIA in 16 (29.1\%), PsA-like in 6 (10.9\%), PMR-like in $5(9.1 \%)$ among others. Time from $\mathrm{ICl}$ to irAEs was $8.3 \pm 8.4$ months $(\mathrm{mo})$. irAE presented earlier in patients with combined $\mathrm{ICI}$ therapy than in patients with monotherapy $(6.5 \pm 4.0$ vs $8.6 \pm 8.9 \mathrm{mo}, \mathrm{p}=\mathrm{NS}$, Figure 1A). Time (in mo) from $\mathrm{ICl}$ initiation to irAE onset was different according to treatments. For Nivolumab $10.0 \pm 10.6$, Anti TIM3 $10.0 \pm 1.4$, Durvalumab 9.0 \pm 2.0 , Ipilimumab 7.98 \pm 9.21 , Pembrolizumab $7.28 \pm 7.53$, Avelumab 6.0 and Atezolizumab $4.4 \pm 5.38 \mathrm{mo}$ (Figure 1B). Time from $\mathrm{ICl}$ initiation and onset also differs among rheumatic irAEs (Figure 2). Mean time follow-up was 13.4 $\pm 10.9 \mathrm{mo}$. At the last visit, $45 \%$ were under GC, mean dose of $3.6 \mathrm{mg} / \mathrm{d}$ (range $0-40$ ). DMARD were needed in $15 \%$ of patients (6 patients MTX, 1 with LEF and $1 \mathrm{SFZ}$ ). At the last visit, 11 (22.9\%) patients remain with persistent arthritis, $25 \%$ intermittent flares and $52 \%$ had a self-limited pattern. Regarding oncologic outcome, $30.2 \%$ were on remission, $30.2 \%$ in partial response and $39.6 \%$ with tumor progression. Eleven (20\%) of patients died.

Conclusion: We described different clinical patterns according treatment and irAEs. Combined ICI therapy and patients treated with Atezolizumab had earlie onset of symptoms. Vasculitis and PMR-like syndromes appear in earlier phases. After a mean follow-up of around 1 year, one-quarter of the patients remain with persistent arthritis and $15 \%$ require DMARD therapy.

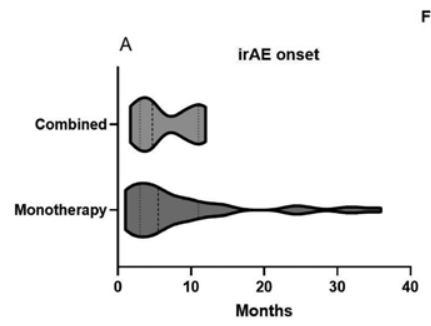

Figure 1

Figure 2

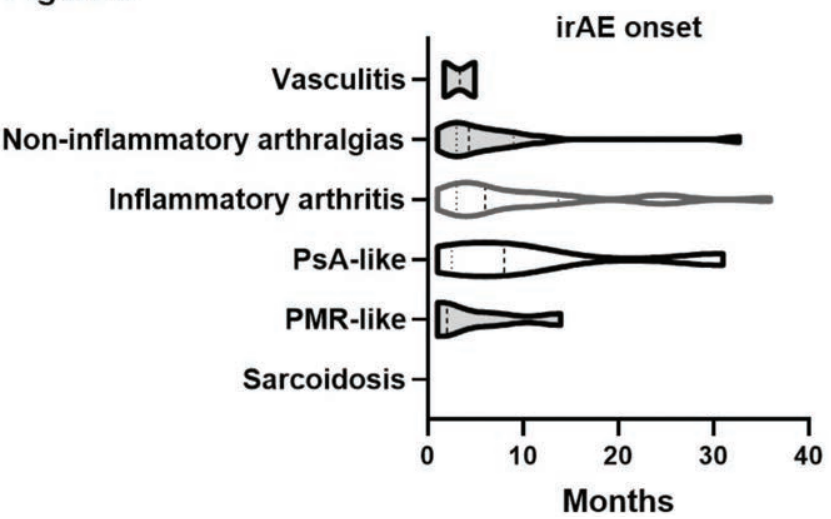

Disclosure of Interests: Jose A. Gómez-Puerta Speakers bureau: Abbvie, BMS, GSK, Lilly, Pfizer, Roche, Carolina Perez-Garcia: None declared, David Lobo Prat: None declared, Roberto Gumucio: None declared, Fabiola Ojeda: None declared, Ana Milena Millán Arciniegas: None declared, Sebastian Rodriguez Garcia: None declared, Virginia Ruiz Speakers bureau: Lilly, Pfizer, Héctor Corominas Speakers bureau: Abbvie, Lilly, Pfizer, Roche DOI: 10.1136/annrheumdis-2020-eular.4308

\section{OP0277 \\ AURORA PHASE 3 STUDY DEMONSTRATES VOCLOSPORIN STATISTICAL SUPERIORITY OVER STANDARD OF CARE IN LUPUS NEPHRITIS (LN)}

C. Arriens ${ }^{1,2}$, S. Polyakova ${ }^{3}$, I. Adzerikho ${ }^{4}$, S. Randhawa ${ }^{5}$, N. Solomons ${ }^{5} .{ }^{1}$ Oklahoma Medical Research Foundation, Oklahoma City, United States of America; ${ }^{2}$ University of Oklahoma Health Sciences Center, Oklahoma City, United States of America; ${ }^{3}$ LLC Medical Center, Kemerovo, Russian Federation; ${ }^{4}$ Minsk Regional Clinical Hospital, Minsk, Belarus; ${ }^{5}$ Aurinia Pharmaceuticals, Victoria, Canada

Background: Voclosporin (VCS) is a novel high potency calcineurin inhibito (CNI) with a favorable metabolic profile and a consistent predictable dose response potentially eliminating the need for therapeutic drug monitoring LN occurs more frequently and is more severe in Hispanic/Latino ethnicity SLE patients. The recently completed phase 3 AURORA study builds on the favorable efficacy seen in the Phase IIb AURA-LV study in patients with active LN.

Objectives: Document efficacy and safety of VCS vs placebo over one year when used with 2 grams of MMF daily and a rapid steroid taper in patients with active LN. Methods: AURORA is a Phase III multicenter, randomized, double-blind, placebo-controlled 52-week study of active LN patients. Patients were randomized 1:1 to VCS (23.7 mg BID) or placebo in combination with mycophenolate (MMF, $1 \mathrm{~g}$ $\mathrm{BID}$ ) and rapidly tapered oral steroids. The primary endpoint was renal response (RR) at 52 weeks, defined as UPCR of $\leq 0.5 \mathrm{mg} / \mathrm{mg}$, eGFR $\geq 60 \mathrm{~mL} / \mathrm{min}$, or no confirmed decrease from baseline in eGFR of $>20 \%$, presence of sustained, low dose steroids and no administration of rescue medication. Ethnicity subgroup analyses of RR was also undertaken given the higher severity of disease in the Hispanic/Latino LN patients.

Results: There were 357 patients enrolled, $88 \%$ female, median age of 31 and $33 \%$ of Hispanic/Latino ethnicity. Renal response by intention to 